\title{
Peculiar lightning-related events observed by the surface detector of the Pierre Auger Observatory
}

\author{
Roberta Colalillo $^{* a}$ for the Pierre Auger Collaboration ${ }^{b}$ \\ ${ }^{a}$ Università Federico II di Napoli and INFN, Sezione di Napoli, Italy \\ ${ }^{b}$ Observatorio Pierre Auger, Av. San Martín Norte 304, 5613 Malargüe, Argentina \\ E-mail: auger_spokespersons@fnal.gov \\ Full author list: http://www.auger.org/archive/authors_icrc_2017.html
}

\begin{abstract}
Some very peculiar events have been recorded using the surface detector (SD) of the Pierre Auger Observatory. The signals produced by these events in the SD stations last about 100 times more than those produced by cosmic rays. For many events, the number of stations having a long signal is big: for the largest events it goes up to 46 stations. The long-lasting stations are arranged in a circular shape with a radius of about $6 \mathrm{~km}$. Moreover, a correlation with lightning was observed. Many checks were performed to evaluate the possibility that electronics problems could generate the observed signals, but these checks revealed that the peculiar events are indeed produced by particles and/or photons that cross the Auger Cherenkov detectors. A search algorithm was implemented based on the duration of the signal. These events are very sporadic. Using a sample of ten "golden" events, studies are under way to characterize these events, and to understand their origin. The main features of these events will be described and a preliminary reconstruction will be shown.
\end{abstract}

35th International Cosmic Ray Conference - ICRC2017

10-20 July, 2017

Bexco, Busan, Korea

${ }^{*}$ Speaker. 

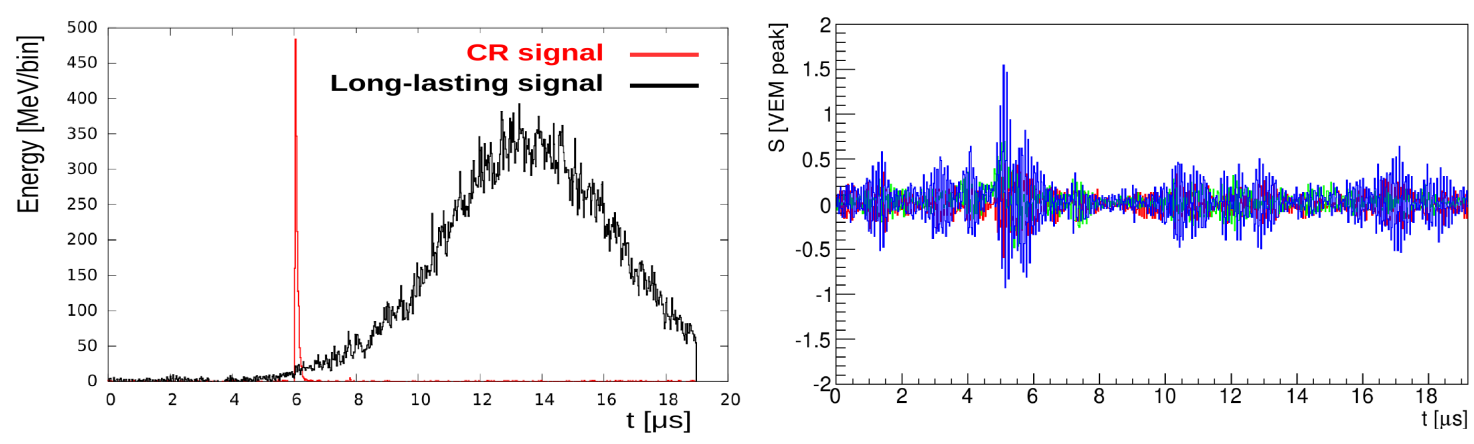

Figure 1: Left: differences between a cosmic-ray trace and the long-lasting signal detected by several triggered stations of the obsereved events. Right: signal collected by a so-called lightning station. Each color corresponds to the signal from one of the three PMTs.

\section{Introduction}

Peculiar events have been collected by the surface detector (SD) of the Pierre Auger Observatory. These events are characterized by SD stations with a long-lasting signal compared to the standard Auger signal produced by extensive air showers, and by stations collecting a signal dominated by high frequency noise. Moreover, for the events with a high number of stations, the long-signal stations are arranged in a circular shape with a radius of about $6 \mathrm{~km}$. The reality of these events was checked, an algorithm for their search was developed, and several studies are in progress to understand their main characteristics and their origin.

The Pierre Auger Observatory is located in the Argentinian Pampa at about $1400 \mathrm{~m}$ a.s.l. It is a hybrid instrument composed of a fluorescence detector (FD), which studies the longitudinal profile of the cosmic-ray showers, and of a SD array which maps the distribution of shower particles at the ground [1]. The SD is made up of 1600 water-Cherenkov detectors (WCDs) placed on a triangular grid of $1500 \mathrm{~m}$ spacing which covers an area of $3000 \mathrm{~km}^{2}$. Each WCD consists of a $3.6 \mathrm{~m}$ polyethylene tank containing a liner with a reflective inner surface and filled with 12,000 litres of ultra-pure water. Cherenkov light produced by the passage of relativistic charged particles through the water is collected by three PMTs. The two output signals from PMTs, one directly from the anode (HG channel) and the other one from the last dynode (LG channel) with an amplification factor of 32 to increase the dynamic range, are processed by six FADCs with a sampling rate of $40 \mathrm{MHz}$.

\section{Main Features}

The first peculiar event was recorded on 4 May 2005 at about $4 \mathrm{am}$. It triggered 65 stations. It differs from cosmic-ray events in the time-scale and the spatial distributions of the WCDs involved. Among the event stations, there are 24 stations with signals that last for a long time (long-signal stations). In Fig. 1-left, this signal, that occurs over $\sim 10 \mu \mathrm{s}$, is compared to a cosmic-ray signal lasting $\sim 0.1 \mu \mathrm{s}$. Another important characteristic is the presence of at least one active station with high frequency noise, called a lightning station, shown in Fig. 1-right. Also stations with a standard cosmic-ray trace are present in some of these events, but they are not correlated in time with the 

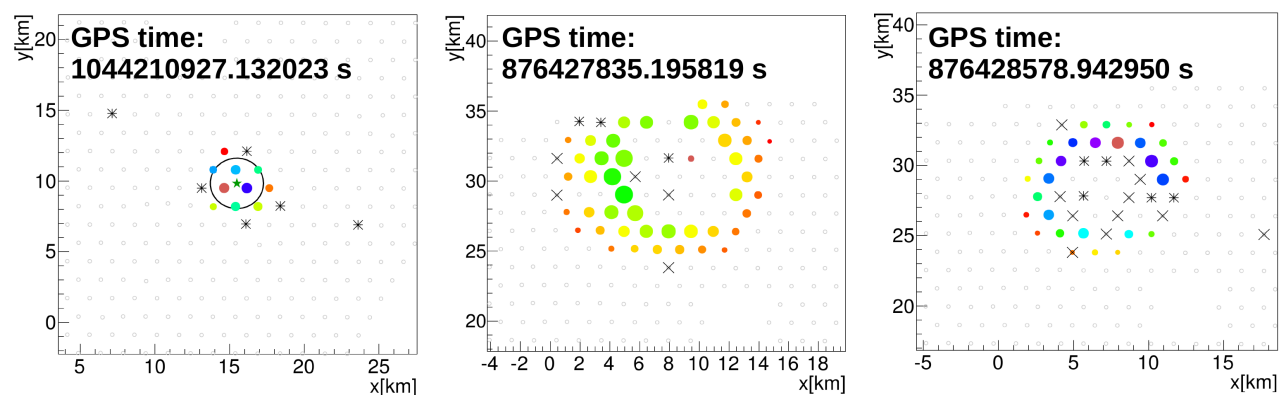

Figure 2: Footprint of three events at the ground. The size of the colored markers (long-signal stations) is proportional to the signal in the stations, the color is related to the arrival time. The blue stations arrive first, finally the red ones. The stars are the lightning stations, while the crosses are the standard cosmic-ray stations. Only the last two events, which have a bigger number of stations, present a depletion of the signal in the center, but the main characteristics of the three events are the same. For example, the long-signal stations are always organized in a circular shape as highlighted by the circumference superimposed on the stations in the first event.

long-signal stations. The footprint of three events at the ground is shown in Fig. 2. The longsignal stations, represented by the coloured markers, are organized in a circular shape. The stars symbolize the lightning stations. Crosses indicate the stations with cosmic-ray traces. The footprint of the first event on the left has a radius of $\sim 2 \mathrm{~km}$, the other two events have a bigger number of long-signal stations, a radius of $\sim 6 \mathrm{~km}$, and present a depletion of the signal in the center. The signal and timing characteristics of these three events are the same.

\section{Checks for the Reality of the Observed Events}

These events are very peculiar. Therefore, it is very important to test that the detected signals are really compatible with Cherenkov light produced inside the stations, and are not artificially induced by electromagnetic noise. We checked the consistency among the signals recorded by the three PMTs and that the HG and LG signal for each PMT are in a consistent ratio. The three PMT signals are shown in Fig. 3-left in three different colors, red, blue, and green, together with the ratios between two PMTs at a time calculated bin-by-bin, and the distributions of the mean of these ratios from all stations, that are peaked at 1 as we expected.

The HG and LG signals are shown in red and blue respectively in Fig. 3-right. When the high gain channel is not saturated and on the low gain channel there is a signal with an amplitude not too low, the ratio between the high gain and the low gain signal multiplied by the amplification factor (D/A constant) is expected to be 1 . To verify this, the ratio was computed for each station with a LG signal with an amplitude not too low (at least 5 bins that are above 7 FADC counts are requested), excluding the saturated part of the HG channel. The distributions obtained for each PMT following these cuts are also shown in figure 3, and they are peaked at 1 . The cut on the LG signal is very strict, but we still found six events with a big number of long-lasting stations. These events are physical: they are not due to electromagnetic noise. In the following, these cuts will be released, and the HG signal will be used except in the cases where the HG channel is saturated. Another important characteristic that has to be checked is the depletion of the signal in the center of large events. We do not know if it is physical. The SD trigger, optimized for cosmic rays, may not be adequate to handle the complex timing of these events. Work is being done at the station 


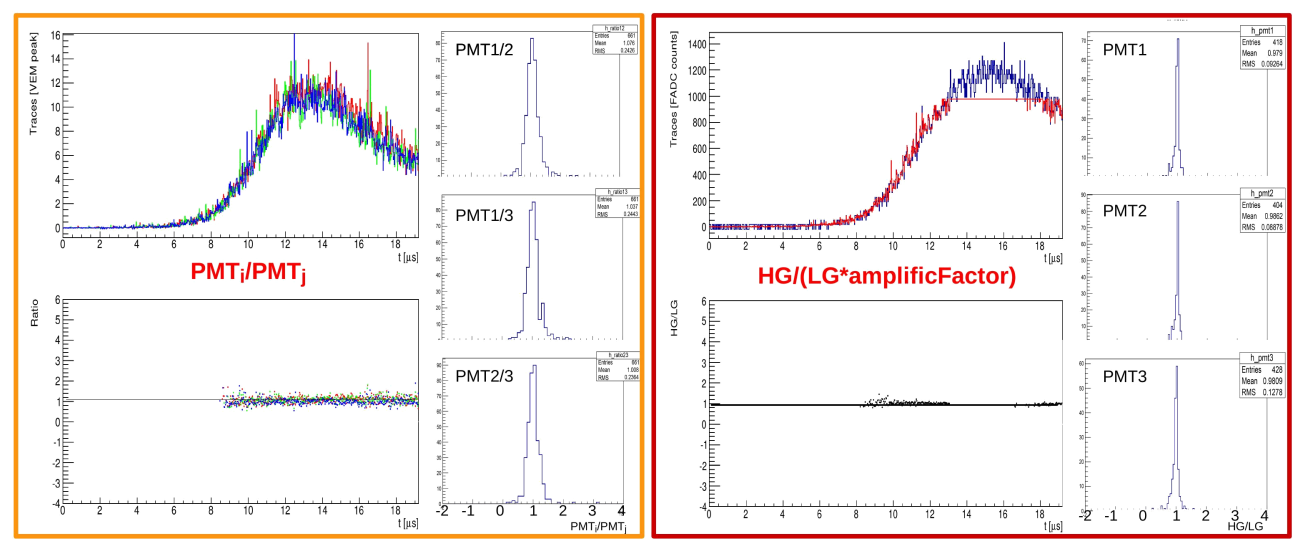

Figure 3: Left: HG signal collected by the three PMTs of an active station of one of the detected events (left-top). Ratios between two PMTs at a time calculated bin-by-bin (left-bottom). Distributions of the mean of the ratios that are peaked at 1 as expected (right). Right: HG and LG signal collected by one PMT in red and blue respectively (right-top). HG signal divided by LG signal multiplied by the amplification factor (right-bottom). Distributions of the mean of the ratio obtained using all stations which pass the cuts described in the text.

trigger level to explore if the central data of events with many stations may be lost due to the high frequency signal detected in the central stations before our events.

\section{Search Algorithm}

The data sample analyzed in this work consists of all events collected by the SD from January 2004 to 15 May 2017, that pass the first standard Auger trigger levels. This cut is looser than the one required for the standard SD analyses [2]. A first cut is applied on data to select events with at least one lightning station. It was fixed after the observation, on a large sample of data, our peculiar events containing both long-signal stations and lightning stations. On selected events, about 200,000, a "moving mean" on 9 bins of the HG trace is performed to reduce fluctuations. On this smeared trace, at least 80 consecutive bins above 1 baseline error are requested (long-signal condition); if there are at least 10 stations with at least two PMTs with a long signal, the event is accepted. Applying this algorithm, 105 events were selected. Among them, there is a large number of events with noisy stations or several consecutive muon peaks confused with long-lasting stations. A visual scan selected 28 events with actual long-lasting stations. 16 of these events have a large number of long-lasting stations, arranged in a ring shape with a big radius. Most of the selected events were collected between the end of 2007 and the beginning of 2008. They were detected both during the day and the night. Most of our events are concentrated in few days. In particular, four events were collected in 15 minutes: three of them happened in $1.5 \mathrm{~ms}$ and in the same zone of the array. They are shown in figure 4. Unfortunately, in 2007, the array was not completed, but long signal and lightning stations are dominant in these events.

The high frequency noise observed in several stations could be associated with lightningcaused signal, which suggests that these events happened during thunderstorms. To test this hypothesis, we checked the correlation between our events and lightning strikes as recorded by the WWLLN (World Wide Lightning Location Network) catalogue. We compared 22 events and we found a correlation for 9 events, $\sim 41 \%$ of our sample. The time difference between our events and 

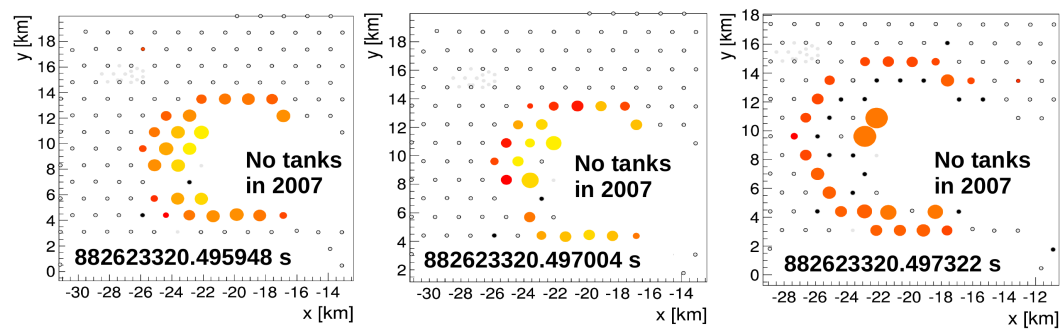

Figure 4: Three events collected in $1.5 \mathrm{~ms}$ in the same zone of the array.
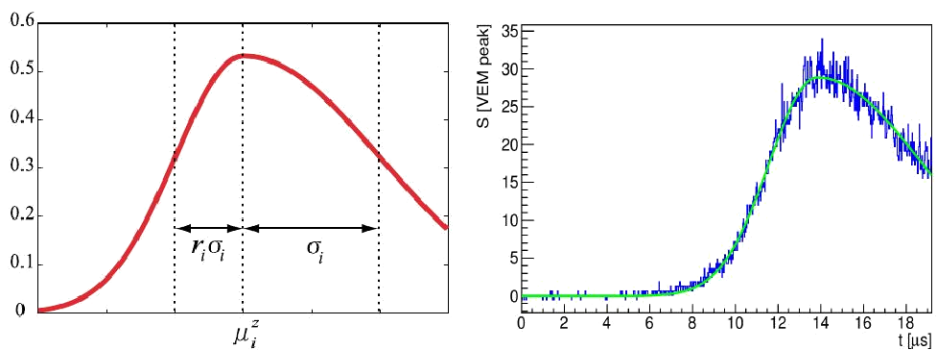

Figure 5: The asymmetric Gaussian is the function which best describes our signal shape (left). Fit obtained from an unweighted chi-square minimization using the asymmetric function. In this case, the low gain signal (blue) is used because the high gain channel is saturated (right).

WWLLN data spans from $10 \mu$ s to $100 \mathrm{~ms}$. The spatial correlation is very good considering that the global location accuracy for WWLLN network assuming 5-station involvement ranges over 1.9 to $19 \mathrm{~km}$ [3]. Five lightning stations were installed at the Observatory by the AERA group [4]. A more detailed correlation study can be performed in future. Several experiments, on satellite [5] and at ground [6][7], have claimed the observation of radiation bursts in coincidence with lightning activity. The relationship between the observed $\gamma$ emission and lightning is under investigation. Further studies on our events are necessary to understand if we are observing the same radiation bursts or new phenomena.

\section{Long-lasting Signal Characteristics}

In many cases, the long-lasting signals of the events are not fully contained in our acquisition time window. To recover the part of the signal we cannot detect directly and the saturated part when also the LG channel is saturated, we need to characterize in the best way the shape of the signal as follows. We used the small sample of stations where the signal is completely visible to choose the fit function. The asymmetric Gaussian distribution best describes the leading and falling edge of the signal. It is shown in Fig. 5 together with an example of fit. A chi-square minimization is performed to fit the signal. To define the common weight to assign to each bin of the trace, we performed the Fourier transform of the long-lasting signal and separated the signal frequencies from the fluctuations. The inverse transform of the signal in the frequency domain with frequencies bigger than $2 \mathrm{MHz}$ is shown in Fig. 6-left. The RMS of this signal, consistent with the Poissonian fluctuations, is used as weight. To fit all the parameters of the asymmetric Gaussian, we need to see at least a small part of the falling edge of the signal. For this reason, we require that the peak 

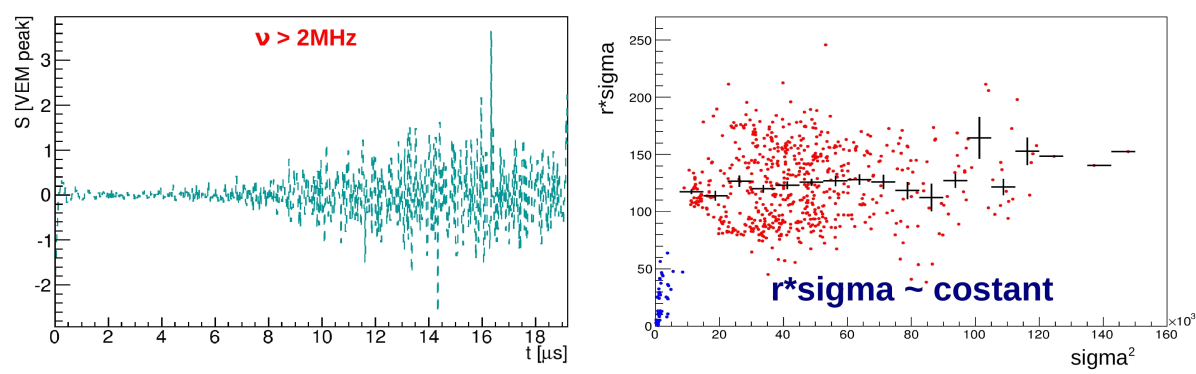

Figure 6: Left: Inverse transform of the long-lasting signal in the frequency domain after cutting the frequencies less than $2 \mathrm{MHz}$ corresponding to the signal. The RMS of the Inverse transform is used as weight for the signal fit. Right: Correlation between $r_{i} \sigma_{i}$ and $\sigma_{i}^{2}$ obtained from the fit of "good" stations: $r_{i} \sigma_{i}$ is constant as a function of $\sigma_{i}^{2}$.
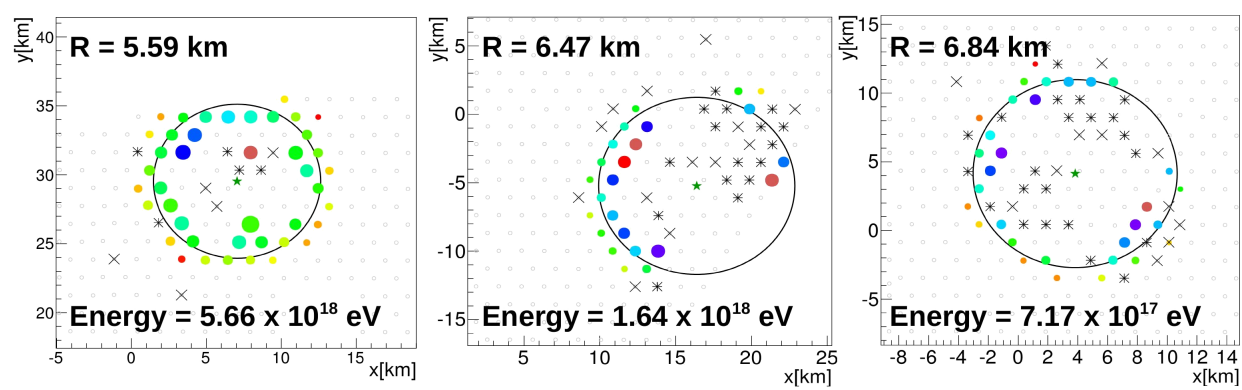

Figure 7: First reconstruction steps for some events.

of the Gaussian is in the DAQ window. We select good fits requiring that the percentage difference between the sum of the content of the trace bins and the integral of the fitting function in our time window is less than $5 \%$ and that the duration of the total fitting function is less than $100 \mu$ s. Stations where at least two of the three PMT signals meet these conditions will be called "good" stations. From the distributions and correlations among the fit parameters of "good" stations, we observed that the rise time of the signal $\left(r_{i} \sigma_{i}\right)$ is smaller than the fall time $\left(\sigma_{i}\right), \sigma_{i}$ is bigger than $2.5 \mu$, and $r_{i} \sigma_{i}$ is constant as a function of $\sigma_{i}^{2}$ (see Fig. 6-right).

The signal detected by each PMT is given by the total integral of the fitting function. The signal per station is the mean of the PMT signals. The reconstruction will be performed only for those events with at least eight "good" stations. Ten events pass this cut, and the $\sim 80 \%$ of this sample shows a correlation with WWLLN lightning.

\section{Event Characterization: Reconstruction and Global Features}

The first step to study the global characteristics of our events is to reconstruct the center of their circular footprint. An unweighted chi square minimization allows us to find the center (green star) and the radius of the circle shown in Fig. 7 for some events.

The energy deposited in each long-signal station spans from $\sim 10^{4} \mathrm{MeV}$ to $\sim 10^{6} \mathrm{MeV}$. Knowing this energy and the number of stations, we can calculate the average energy per $\mathrm{m}^{2}$ and multiply it for the area really covered by the long-lasting stations to obtain the total deposited energy of the event. The energies oscillate between $10^{17}$ and $10^{18} \mathrm{eV}$. The energy deposited at ground by a vertical cosmic-ray shower initiated by a proton with energy $10^{19} \mathrm{eV}$ is about two orders of magnitude 

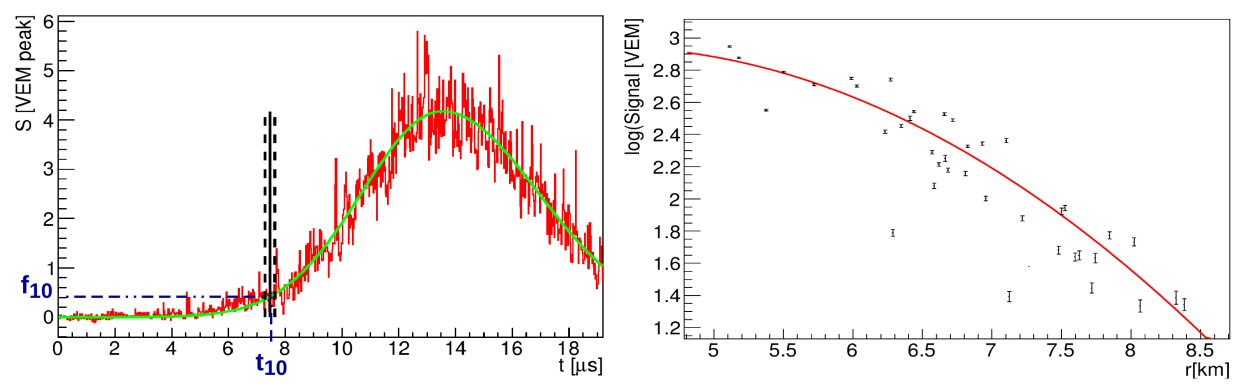

Figure 8: Left: Start time of long-lasting signal: $10 \%$ of the value of the asymmetric Gaussian in its peak. Right: $\log ($ long signal) as a function of the distance from the center. It is larger in the inner stations and decreases as the distance from the center increases.

lower. Moreover, comparing our energy per $\mathrm{m}^{2}$ with the deposited energy at ground measured at $100 \mathrm{~m}$ from the source in NaI scintillators by Dwyer et al. [6], we observed that our energy density is of the same order of magnitude or bigger close to the center of the circle (at least $0.5 \mathrm{~km}$ from the center), lower at the outer region. The energy measured using plastic scintillators of the TA experiment [7], instead, is $\sim 10^{2} \mathrm{MeV}$ per $\mathrm{m}^{2}$, two orders of magnitude lower than our smallest energy, but it is important to remember that they have a small efficiency for photon detection.

The start time of our long-lasting signals is defined as the time corresponding to the $10 \%$ of the value of the asymmetric Gaussian in its peak, $t_{10}$. It is depicted (black line) with its uncertainty (black dashed lines) in Fig. 8-left. The time of each long-lasting station is given by the GPS time of the station plus $t_{10}$. The station time as a function of the distance from the center is shown in Fig. 9. The signal arrives first in the inner stations and then reaches the external ones. The log(long signal) as a function of the distance from the center, instead, is shown in Fig. 8-right and is well fit by a parabola. The signal is larger in the inner stations and decreases as the distance from the center increases.

The arrival times of the long-signal stations are fit considering a spherical propagation model. Four parameters are obtained: $x_{0}, y_{0}$, and $c t_{0}$, that are the coordinates of the origin point of the event, and $t_{\text {off }}$ which takes into account the offset between the event time and $t_{0}$. The altitudes of the origin point obtained from the spherical fit are very low, do not exceed $1 \mathrm{~km}$. To compare the evolution of the front with expectations, a simple MC assuming a spherical front was performed. The origin point of the event was fixed at different altitudes, for each impact point at ground, the distance from the origin point was calculated, and assuming relativistic particles, the arrival time was obtained. The simulated arrival times are represented by the colored lines shown in Fig. 9. Each line corresponds to a different altitude of the origin point of the event, from 0 to $10 \mathrm{~km}$. Subtracting $t_{\text {off }}$ from the measured arrival times, they superimpose to the simulated line corresponding to an origin point at $0 \mathrm{~km}$ as we can see in Fig. 9 for two events. The event without depletion of the signal in the center shown on the left of Fig. 2 do not pass the quality cuts asked for the reconstruction because the peak of the long-lasting signals is never visible in our time window. Anyway, performing a raw reconstruction of this event, we observe also in this case that the signal arrives first in the inner stations, where it is bigger than in the outer stations, and that the propagation of this event is compatible with a spherical front with the source very close to ground and a radial expansion at the speed of light. 

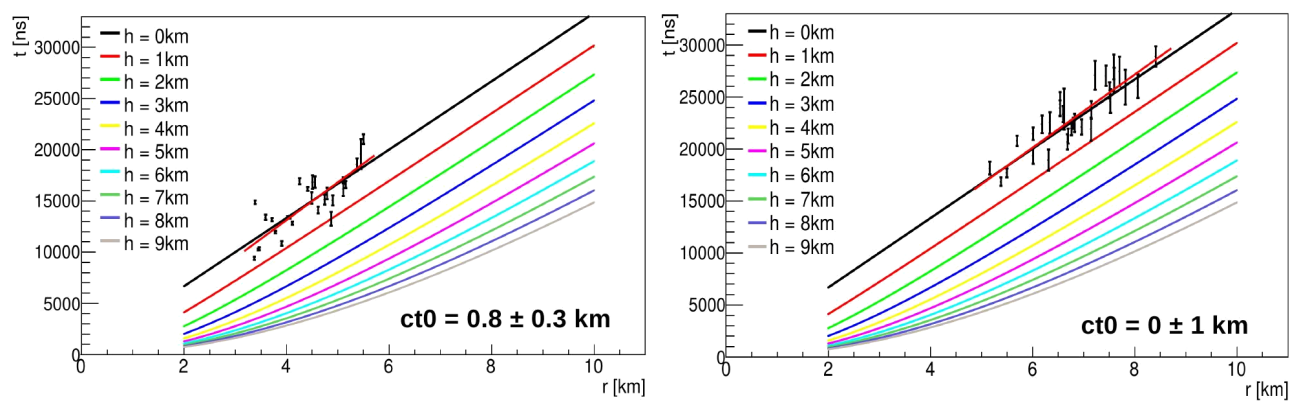

Figure 9: Arrival times as a function of the distance from the center. The black points are the real arrival times of the long stations with their uncertainty. The colored lines represent the simulated times obtained by a simple MC. Each line corresponds to a different altitude of the origin point of the event, from 0 to $10 \mathrm{~km}$. Data are compatible with an origin point of the event very close to ground.

\section{Conclusions}

Very peculiar events, characterized by the presence of stations with very long-lasting signals have been detected by the Pierre Auger Observatory. Some of them have many active detectors arranged in a ring shape, with no detected signal at the center. Events with many stations were characterized. Their signals are well described by an asymmetric Gaussian shape with the rising edge steeper than the trailing one. A correlation between the two widths was observed. The amplitude of the signal is bigger in the inner part of the ring and decreases with the increasing of the distance from the center. The event moves from the center of the circle to the external part, the observed timing is consistent with a spherical front expanding at the speed of light with an origin point very close to ground or to a cylindrical front. For many events, a correlation with lightning was observed. Many experiments have collected radiation bursts in coincidence with lightning activity. Studies are in progress to understand if we are also observing radiation bursts or other phenomena and if the depletion of the signal observed in some big events is physical or not.

\section{Acknowledgments}

The authors wish to thank the World Wide Lightning Location Network (wwlln.net), a collaboration among over 50 universities and institutions, for providing the lightning location data used in this paper.

\section{References}

[1] Pierre Auger Collaboration, Nucl. Instrum. Meth. A 798 (2015) 172-213.

[2] Pierre Auger Collaboration, Nucl. Instrum. Meth. A 613 (2010) 29-39.

[3] C.J. Rodger, J.B. Brundell, and R.L. Dowden, Ann. Geophys. 23 (2005) 277-290.

[4] J. Rautenberg, for the Pierre Auger Collaboration, 34th Int. Cosmic Ray Conf., The Hague (2015).

[5] G.J. Fishman et al., Science 264 (1994) 1313-1316.

[6] J.R. Dwyer et al., Geophys. Res. Lett. 31 (2004) L05119.

[7] Telescope Array Collaboration, arXiv:1705.06258. 